\title{
Hypercholesterolemia Increases the Production of Leukotriene B4 in Neutrophils by Enhancing the Nuclear Localization of 5-Lipoxygenase
}

\author{
Xiao-Feng Lai ${ }^{a, c}$ Hua-Di Qin ${ }^{a}$ Lei-Lei Guo b,d Zi-Guo Luo ${ }^{b}$ Jing Changa \\ Chun-Chang Qin ${ }^{a}$ \\ aCardiology Department, The First Affiliated Hospital of Chongqing Medical University, ${ }^{\mathrm{b} C o l l e g e}$ of \\ Basic Medicine, Chongqing Medical University, 'Cardiology Department, ChongGang General Hospital, \\ Chongqing, China; 'Cardiology Department, JiangYou People's Hospital, JiangYou, SiChuan, China
}

\section{Key Words}

5-Lipoxygenase • Leukotriene B4 • Hypercholesterolemia

\begin{abstract}
Aims: Neutrophils can synthesize leukotriene B4 (LTB4) by activating the 5-lipoxygenase (5-LO)signaling pathway. LTB4 is a pro-inflammatory mediator associated with the etiology and progression of atherosclerosis. It can increase function and number of neutrophils in an autocrine manner. Since hypercholesterolemia is associated with an increase in the number and function of neutrophils, we hypothesized that this effect could be mediated through increased production of LTB4 in neutrophils. Methods/Results: Hypercholesterolemia was modeled in Wistar rats by feeding them with a high cholesterol diet. The induction of hypercholesterolemia caused an increase in the plasma levels of LTB4, following lipopolysaccharide stimulation. This effect was recapitulated in vitro, both in the presence and absence of stimulation with the activator of 5-LO, A23187. Neutrophils in hypercholesterolemia rats expressed similar total levels of 5-LO as control rats, but displayed increased nuclear localization of 5-LO, as well as elevated levels of phosphorylated 5-LO and ERK1/2. In vitro, M $3 C D / c h o l e s t e r o l$ complexes enriched cholesterol in neutrophils, resulted in similar changes in 5-LO/LTB4. In addition, these alterations could be inhibited with the ERK inhibitor PD98059. Conclusion: Hypercholesterolemia increases LTB4 production in neutrophils by increasing the nuclear localization of 5-LO, which is the result of its phosphorylation by activated ERK1/2.
\end{abstract}

X.-F. Lai, H.-D. Qin and L.-L. Guo contributed equally to this manuscript.

Chun Chang Qin, MD

Cardiology Department, The First Affiliated Hospital of Chongqing Medical University, YouYi Road 1\#, Chongqing 400017 (China)

Tel. +86 15025450166, Fax 86 +2389011430, E-Mail QCC3011@hotmail.com 


\section{Introduction}

The contribution of monocytes to the pathology and progression of atherosclerosis has been established. However, the role of neutrophils, the most abundant leukocytes in circulation, has not been extensively investigated. Recent reports revealed that neutrophils are proinflammatory cells that might be associated with promoting an atherosclerotic condition [1]. Specifically, neutrophils and the proteinases secreted by them have been detected in plaques. Other studies have shown that hypercholesterolemia (HC) can induce neutrophilia (an increase in cell number), while the degree of this expansion is positively correlated with the extent of formation of early atherosclerotic lesions [2]. It is speculated that the increased levels of membrane cholesterol characteristic with the aforementioned conditions could enhance the adhesive properties of neutrophils, leading to increased possibility of membrane tethering and whole cell deformation [3]. Neutrophils isolated from HC patients display increased production of superoxide and increased adhesion to endothelial cells. Furthermore, in mice that were fed a high-cholesterol diet, the adhesive and migratory properties of neutrophils were found to be elevated.

Neutrophils are known to produce lipid mediators such as the potent chemoattractant leukotriene B4 (LTB4) [1]. Recent human genetic and population studies, together with complementary animal studies, have suggested that LTB4 promotes atherosclerosis by mediating increased leukocyte chemotaxis and vascular inflammation [4]. LTB4 has been detected in atherosclerotic lesions, and its levels have been correlated with the degree of leukocyte infiltration in atherosclerotic plaques. In LDLr-/- mice (knockouts of low density lipoprotein receptor) fed with a high fat diet, blockade of the LTB4 receptor reduced the infiltration of vascular lesions by monocytes [5]. Others have demonstrated that the leukotriene transcriptional pathway contributes to early vascular remodeling during atherosclerosis [6].

The synthesis of LTB4 from arachidonic acid requires the function of a key regulatory enzyme, 5-Lipoxygenase (5-LO). 5-LO plays a pivotal role in LPS (lipopolysaccharide)mediated adhesion of monocytes to the vascular endothelium by inducing increased expression of Mac-1 on the cell surface [7]. Cipollone et al found that the expression of 5-LO is increased in the carotid plaques of patients who have a history of ischemic conditions [8]. Evidence from clinical studies and animal models suggests that 5-LO is a promising target for therapeutic strategies aimed at reducing inflammation in atherosclerotic plaques. For example, in patients diagnosed with acute coronary syndrome, a twelve week treatment of the 5-LO inhibitor VIA-2291, reduces systemic LTB4 production that could reduce the severity of atherosclerotic lesions in these patients [9]. The synthesis of LTB4 in neutrophils is also dictated by the availability of free arachidonic acid (AA) and the subcellular location of 5-LO. In resting neutrophils, 5-LO is localized to the cytoplasm. However, once activated, it is redistributed to the nuclear membrane to initiate the biosynthesis of LTB4. The activation and subsequent nuclear localization of 5-LO is mediated by phosphorylation of its C-terminal catalytic domain (p-5-LO) by extracellular signal regulated kinases (ERKs). Interestingly, hypercholesterolemia has been shown to activate ERKs in smooth muscle cells, although the physiological response to high cholesterol levels in the blood can vary depending on the cell type. In this study, we explore the mechanistic association between blood cholesterol levels and the activity of neutrophils. Specifically, we determined whether hypercholesterolemia can increase LTB4 production in rat neutrophils by activating ERK1/2 signaling.

\section{Materials and Methods}

\section{Animal Experiments}

All experiments were conducted in accordance with NIH guidelines on the welfare of experimental animals and with the approval of the Animal Care Committee of ChongQing Medical University. Since gender may affect 5-LO activity [10], we included only female Wistar rats $(n=24 ; 300 \pm 30 \mathrm{~g})$ provided by Chongqing 
Medical University Laboratory Animal Center (Chongqing, PR China). They were randomly divided into two groups (12 rats per group): 1. Control rats were fed with normal rat chow; 2. Hypercholesterolemia group rats were fed with normal rat chow supplemented with cholic acid (1\% wt/wt), cholesterol $(2 \% \mathrm{wt} / \mathrm{wt})$ and cooking oil ( $5 \% \mathrm{wt} / \mathrm{wt}$ ). Rats were housed under standard conditions (temperature $=25 \pm 1{ }^{\circ} \mathrm{C}$, humidity= $60 \pm 10 \%$, and a $12 \mathrm{hr}$ light/dark cycle with light provided from 6 a.m. to 6 p.m.), and free and unlimited access to food and water. After eight weeks, six rats in each group were anesthetized with pentobarbital (40 $\mathrm{mg} / \mathrm{kg}$ injected intraperitoneally (i.p.)), and a sample of their blood was collected. They were then injected with LPS (10 mg/kg i.p.). Six hours later another blood sample was collected. The other six rats in each group were used as the source of neutrophils for all cellular assays. All blood samples were collected by cardiac puncture under anesthesia. The rats were sacrificed by cervical dislocation under anesthesia.

\section{Neutrophil Isolation}

Neutrophils were isolated by centrifuging blood through a Ficoll-Hypaque density gradient (Tian Jin Hao Yang Biological Manufacture Co., China). The purity of the preparation, as determined by Wright's staining of a cytospin sample, was $>95 \%$. Cell viability, as determined by trypan blue exclusion, was $>95 \%$. Neutrophils were stored on ice until use, and were resuspended in either PBS supplemented with $1 \mathrm{mg} / \mathrm{ml}$ glucose and $1 \mathrm{mM} \mathrm{CaCl}_{2}$ (PGC buffer), or RPMI1640 medium without phenol red and serum.

\section{Measurement of lipids and LTB4}

Blood samples were centrifuged at $600 \mathrm{~g}$ for $10 \mathrm{~min}, 4^{\circ} \mathrm{C}$, in order to obtain plasma. The concentrations of total cholesterol (TC), triglycerides (TG), low-density lipoprotein cholesterol (LDL-C) and high-density lipoprotein cholesterol (HDL-C) were determined using colorimetric assays, performed according to manufacturer's instructions (Nan Jing Jiancheng Bioengineering Institute, China). LTB4 levels were estimated using an enzyme immunoassay (EIA) kit (Cayman Chemical, USA), in accordance with the manufacturer's instructions. Sample absorbance at $412 \mathrm{~nm}$ was determined using a Thermo Multiskan MK3 microplate reader, and concentrations of LTB4 were calculated based on a standard curve.

\section{LTB4 Production After Stimulation with A23187}

Neutrophils $\left(5 \times 10^{6}\right)$ were stimulated with an activator of 5-LO, the calcium ionophore A23187 (2.5 $\mathrm{uM}, 37^{\circ} \mathrm{C}$ ) for $30 \mathrm{~min}$ in PGC buffer. The reaction was stopped with $100 \mathrm{ul} \mathrm{methanol} \mathrm{and} 30 \mathrm{ul} 1 \mathrm{~N} \mathrm{HCl}$ on ice. The supernatant was collected and stored at $-80^{\circ} \mathrm{C}$ until analysis.

\section{Indirect immunofluorescence}

Neutrophils $\left(1 \times 10^{5}\right)$ were first allowed to attach to the surface of glass coverslips coated with poly-L-lysine by centrifugation at $30 \mathrm{~g}$ for $1 \mathrm{~min}$. They were then fixed in methanol $\left(-20^{\circ} \mathrm{C}, 30 \mathrm{~min}\right)$ and permeabilized with $0.1 \%$ Tween 20 in PBS (room temperature (RT), $10 \mathrm{~min}$ ), followed by three washes with PBS. Non- specific antibody binding was blocked with $10 \%$ non-immunogenic goat serum for 10 min at RT. Next, the cells were washed with PBS and incubated with polyclonal rabbit anti-5-LO serum 1:1000; Millipore, USA) for $30 \mathrm{~min}$ at RT. The coverslips were then washed 10 times with PBS, incubated with goat anti-rabbit IgG conjugated to Alexa Fluor 488 (1:500; Beyotime Institute of Biotechnology, China) for 10 min in the dark at RT, and washed 10 times with PBS. DNA was stained with $0.1 \mathrm{~g} / \mathrm{ml}$ diamidino-2-phenylindole (DAPI) dissolved in PBS, for 3 min in the dark at RT. Finally, the coverslips were washed 10 times in PBS and mounted on glass slides using an antifade mounting medium (Beyotime Institute of Biotechnology). The fluorescence signal was visualized on a Zeiss LSM 510 confocal scanning microscope.

\section{Measurement of arachidonic acid}

Neutrophils ( $5 \times 10^{7}$ in $1 \mathrm{ml} \mathrm{PGC} \mathrm{buffer)} \mathrm{were} \mathrm{pre-incubated} \mathrm{with} \mathrm{the} \mathrm{12-Lipoxygenase} \mathrm{(12-LO)} \mathrm{inhibitor}$ CDC (10 uM) and the 5-LO inhibitor BWA4C (1 uM), to prevent the conversion of released arachidonic acid to LO metabolites. The reaction was initiated by the addition of either A23187 ( $2.5 \mathrm{uM}$ at $\left.37^{\circ} \mathrm{C}, 30 \mathrm{~min}\right)$ or vehicle (as resting cells), and was terminated using $2 \mathrm{ml}$ methanol and $60 \mathrm{ul} 1 \mathrm{~N} \mathrm{HCl}$, followed by the addition of $2 \mathrm{ml}$ PBS and $60 \mathrm{ug}$ margaric acid (as an internal standard). Released AA was extracted using Agilent SBC18 (4.6 mm $\times 150 \mathrm{~mm}$, 5um) solid phase extraction, and coupled to dimethoxyaniline hydrochloride in the presence of N-ethyl- $\mathrm{N}^{\prime}$-(3-dimethylaminopropyl) carbodiimide. The derivatized AA was then analyzed by reverse phase (RP)- HPLC (SHIMADZU LC - 2010A), and its concentration was measured at $272 \mathrm{~nm}$ [10]. 


\section{Cholesterol enrichment with $M \beta C D /$ cholesterol complexes}

To enrich the cholesterol content in neutrophils, we prepared Methyl- $\beta$-cyclodextrin (M $\beta C D$ ) / cholesterol complexes, as described as before [11]. M $\beta C D$ solution was prepared by dissolving $\mathrm{M} \beta \mathrm{CD}$ (Shanghai Yongye Biological,China)in RPMI1640 medium without phenol red and withoutserum. Cholesterol stock solution was added to a glass tube. The solvent was evaporated, and $5 \mathrm{mmol} / \mathrm{L} \mathrm{M \beta CD}$ was added to the dried cholesterol with the ratio of $\mathrm{M} \beta \mathrm{CD}$ : cholesterol as 4:1. The tube was sonicated for 10 minutes and placed in a shaking incubator overnight at $37^{\circ} \mathrm{C}$. Neutrophils were incubated with vehicle or $\mathrm{M} \beta \mathrm{CD} /$ cholesterol complexes for $30 \mathrm{~min}$, then analyzed using the Amplex Red assay kit (Molecular Probes, USA) to confirm the increase in cholesterol content. In another set of experiments, neutrophils were incubated with either vehicle, $\mathrm{M} \beta \mathrm{CD} /$ cholesterol complexes, or $\mathrm{M} \beta \mathrm{CD} / \mathrm{cholesterol} \mathrm{complexes} \mathrm{supplemented} \mathrm{with} 30 \mathrm{uM}$ ERK inhibitor PD98059 (Promega, USA) for $30 \mathrm{~min}$ at $37^{\circ} \mathrm{C}$. Subcytosolic distribution of 5-LO was detected using Western blot and indirect immunofluorescence microscopy. LTB4 production was examined with an EIA kit as described above.

\section{Fractionation of Neutrophils}

In order to separate neutrophils into different cellular fractions, they were first treated with ice-cold $1 \%$ Nonidet P-40 lysis buffer, vortexed ( 3 x $5 \mathrm{sec}$ ), incubated on ice for $10 \mathrm{~min}$ and centrifuged ( $800 \mathrm{~g}$ for 10 $\min$ at $4^{\circ} \mathrm{C}$ ). The resulting supernatant was comprised of the non-nuclear fractions. The pellet (comprised of nuclear fractions) was resuspended in $500 \mu \mathrm{L}$ ice-cold relaxation buffer. Both fractions were centrifuged again $\left(800 \mathrm{~g}\right.$ for $10 \mathrm{~min}$ at $\left.4^{\circ} \mathrm{C}\right)$. Efficiency of cell lysis and integrity of the nuclei was confirmed by light microscopy using trypan blue to stain dead cells. The nuclear suspension in relaxation buffer was disrupted by sonication ( $3 \times 5$ seconds). Aliquots of both nuclear and nonnuclear fractions were immediately mixed with an equal volume of SDS-b, heated for $6 \mathrm{~min}$ at $95^{\circ} \mathrm{C}$, and analyzed by Western blot for the presence of 5-LO protein [10].

\section{Western Blot}

The total protein extract, prepared by lysing cells with the protein lysis buffer, was separated by SDSPAGE, and transferred to polyvinylidene fluoride membranes. These were then incubated with the following antibodies- polyclonal rabbit anti-5-LO serum (1:1000; Milipore, USA), anti-p-5-LO serum (1:500; Sigma, USA), anti-ERK1/2 serum (1:1000; Cell Signaling Technology, USA), anti-phospho-ERK1/2 (1:1000; Cell Signaling Technology, USA), anti-FLAP (1:500; Santa Cruz Biotechnology, USA). The membranes were then washed and incubated with the horseradish peroxidase-conjugated goat anti-rabbit IgG antibody 1:2,000; Beyotime Institute of Biotechnology). The labeled protein bands were visualized using an ECL Detection System (Beyotime Institute of Biotechnology).The intensity of each band, and hence the level of protein expression, was quantified by densitometric scanning. Data are represented as a ratio of the signal densities of the protein of interest and the corresponding internal control $\beta$-actin or Lamin b.

Statistics

Results are presented as Mean \pm SD. Statistical comparisons were performed using

Student's t-test or paired t-test. $\mathrm{P}<0.05$ was considered significant. All analyses were performed with the statistical software SPSS 11.0.

\section{Result}

Hypercholesterolemia leads to increased LTB4 production in neutrophils

Rats that were fed a high cholesterol diet developed hypercholesterolemia. This was determined by measuring and comparing the plasma levels of LDL-C, TC, HDL-C and TG in control and high cholesterol diet groups (Table1). Since neutrophils are the major source of LTB4, we next determined its level in the plasma in order to assess the activity of neutrophils. Although the basal levels of LTB4 were similar in both groups, following LPS stimulation, LTB4 concentration in the plasma of hypercholesterolemia- induced rats was significantly higher (Fig. 1A). Neutrophils isolated from this group produced significantly more LTB4 in vitro. 
Table 1. Induction of hypercholesterolemia in rats fed with high cholesterol diet. Data are presented are Mean $\pm S D, n=6$; **, $\mathrm{P}<0.01$; Student's t-test

\begin{tabular}{lcccc}
\hline & LDL-C mmol/L & TC mmol/L & TG mmol/L & HDL-C mmol/L \\
\hline Control & $0.91 \pm 0.12$ & $2.37 \pm 0.23$ & $0.79 \pm 0.11$ & $0.95 \pm 0.15$ \\
high cholesterol diet & $1.98 \pm 0.29^{* *}$ & $3.99 \pm 0.32^{* *}$ & $0.86 \pm 0.09$ & $1.05 \pm 0.18$ \\
\hline
\end{tabular}

A
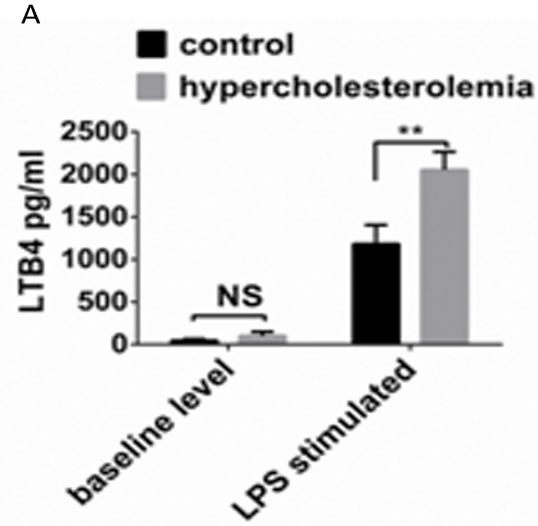

B control hypercholesterolemia

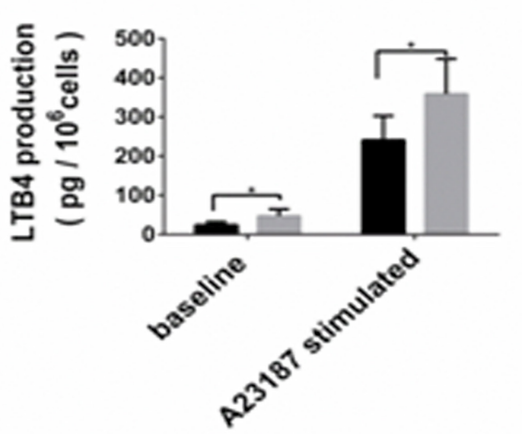

Fig. 1. Hypercholesterolemia increases LTB4 production in neutrophils. (A): The first set of bars represent the plasma LTB4 level in hypercholesterolemia $(89.2 \pm 36.6 \mathrm{pg} / \mathrm{ml})$ and control $(59.6 \pm 29.4 \mathrm{pg} / \mathrm{ml})$ groups without LPS stimulation. The second set of bars represent plasma LTB4 levels after LPS stimulation in hypercholesterolemia $(2052.0 \pm 499.2 \mathrm{pg} / \mathrm{ml})$ and control $(1182.5 \pm 222.7 \mathrm{pg} / \mathrm{ml})$ groups $(\mathrm{p}<0.01)$. (B): The first set of bars represent in vitro LTB4 production in neutrophils isolated from hypercholesterolemiainduced $\left(47.4 \pm 17.3 \mathrm{pg} / 10^{6}\right.$ cells $)$ and control $\left(24.3 \pm 8.4 \mathrm{pg} / 10^{6} \mathrm{cells}\right)$ rats $(\mathrm{p}<0.05)$. The second set of bars represent in vitro LTB4 production in neutrophils isolated from hypercholesterolemia- induced $358.9 \pm 89.5$ $\left.\mathrm{pg} / 10^{6} \mathrm{cells}\right)$ and control $\left(241.2 \pm 61.8 \mathrm{pg} / 10^{6} \mathrm{cells}\right)$ rats ( $\left.\mathrm{p}<0.01\right)$, in response to A23187 stimulation $(2.5 \mathrm{uM}$; $10 \mathrm{~min}, 37^{\circ} \mathrm{C}$ ). Data are presented as Mean $\pm \mathrm{SD}, \mathrm{n}=6$, duplicates; ${ }^{*} \mathrm{P}<0.05$; ${ }^{* *} \mathrm{P}<0.01$; NS: not significant; Student's t-Test.

To determine the association between LTB4 production and activity of 5-LO in neutrophils, we stimulated isolated neutrophils from control and hypercholesterolemia groups with the 5-LO activator A23187. Our assay showed that the synthesis of LTB4 was much higher under a hypercholesterolemic condition, irrespective of A23187 stimulation (Fig. 1B).

Hypercholsterolemia leads to increased nuclear localization of 5-LO in neutrophils

Next, we investigated the cause of increased LTB4 production in the hypercholesterolemia group. It is known that the synthesis of LTB4 is influenced by the following factors: the availability of free AA, level of FLAP (5-Lipoxygenase activating protein), and the activity of 5-LO, LTA4 hydrolase expression levels and activity. First, we measured the release of AA in neutrophils, and found no differences between control and hypercholesterolemia groups (Fig. 2A). Next, we performed Western blot to determine the expression level of FLAP (Fig. 2B) and 5-LO, and again found no differences between the two groups, both with and without A23187 stimulation. Since the activity of 5-LO is dependent not only on the expression level but also its subcellular compartmentalization, we assayed the latter by performing subcellular fractionation of isolated neutrophils [10]. Both nuclear and cytoplasmic fractions were analyzed by Western blot to determine the distribution of 5-LO. We found that the ratio of nuclear to non-nuclear 5-LO was significantly higher in the hypercholesterolemia group when compared to the control group, irrespective of stimulation with A23187 stimulation (Fig. 2C). To further confirm the above results, we immunostained isolated neutrophils and 
Fig. 2. Hypercholsterolemia increases nuclear localization of 5-LO and activity of ERK1/2 in neutrophils. (A): Shown here is the release of AA from neutrophils after A23187 stimulation $(2.5 \mathrm{uM}, 10 \mathrm{~min}$, $37^{\circ} \mathrm{C}$ ), in the hypercholesterolemia and control groups. (B): Western blot analysis of FLAP expression level in the control and hypercholesterolemia groups. (C): Western blot analysis of nuclear 5-LO in neutrophils from hypercholesterolemia and control groups, with and without A23187 stimulation (2.5 M; 5 $\min , 37^{\circ} \mathrm{C}$ ) (D): Indirect immunofluorescent staining of 5-LO in neutrophils of hypercholesterolemia and control groups, with and without A23187 stimulation, ( $n=6$ independent experiments). (E): Western blot analysis of p-5-LO in neutrophils from hypercholeste-
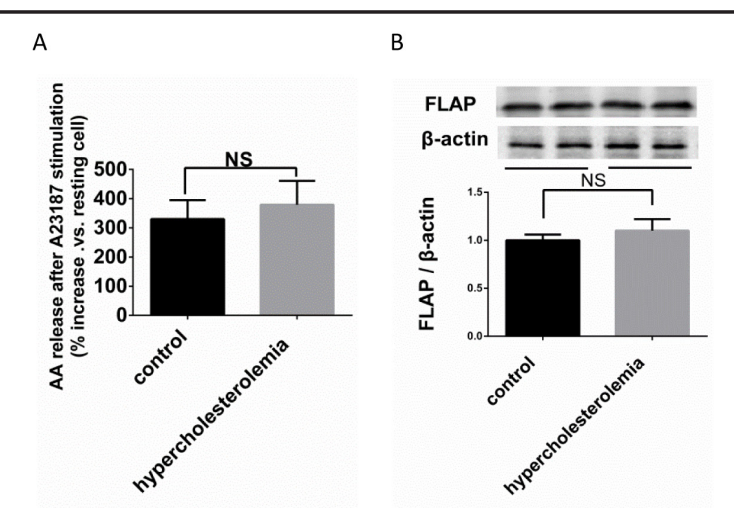

C
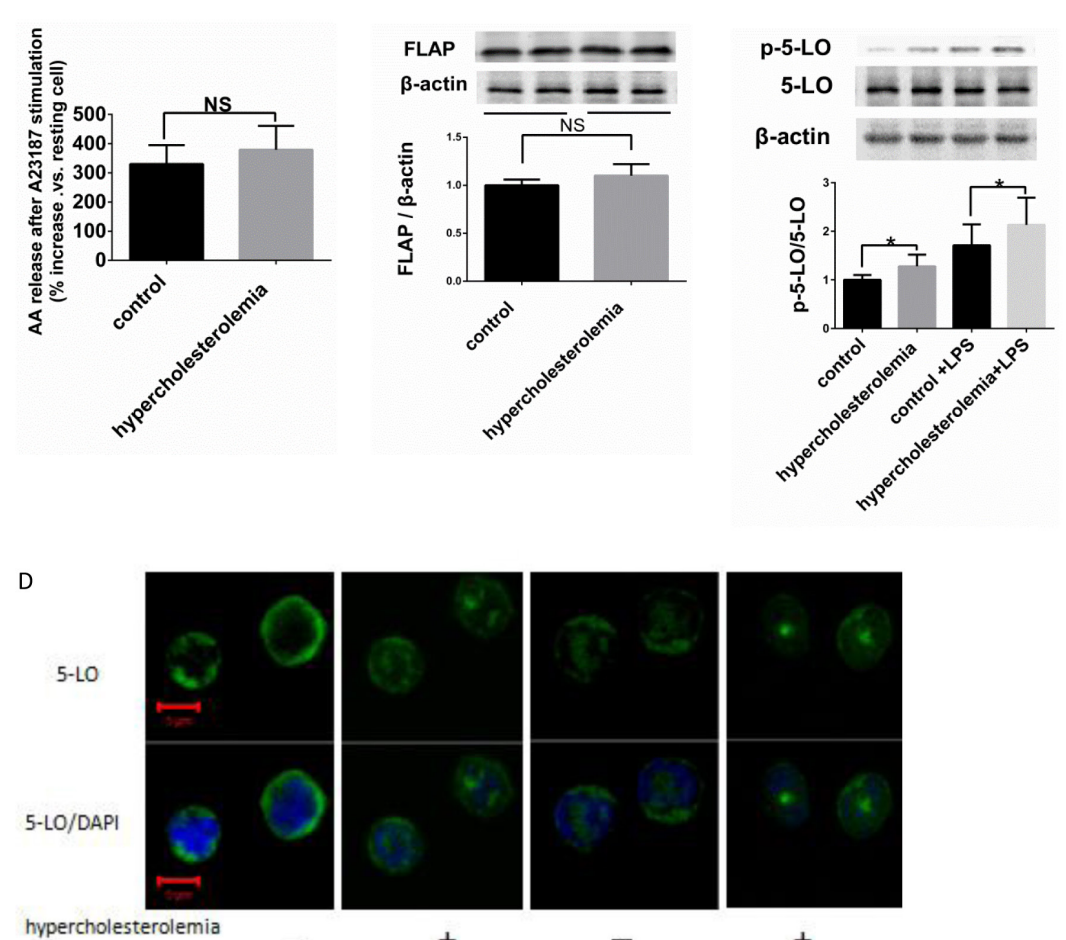

hypercholesterolemia

A 23187

E

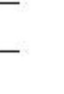
$+$

$+$

F

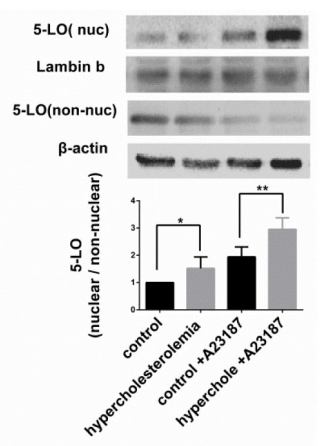

F

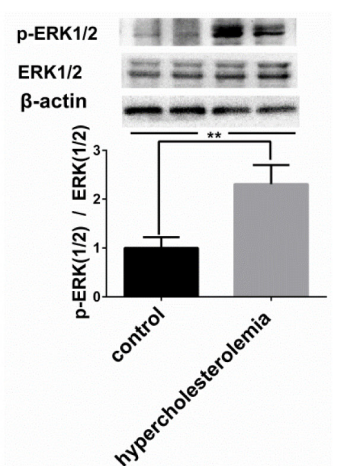

rolemia and control groups, with and without LPS stimulation. (F): Western blot analysis of p-ERK1/2 in neutrophils from hypercholesterolemia and control groups. Data are presented as Mean \pm SD; $n=6$, duplicates. The relative optical density of the control sample was set at $100 \%$. Student's t- test, ${ }^{*} \mathrm{P}<0.05 ;{ }^{* *} \mathrm{P}<$ 0.01 ; NS: not significant.

used indirect immunofluorescence microscopy to examine the subcellular distribution of 5-LO. As expected, we found elevated levels of nuclear 5-LO in the hypercholesterolemia group, both with and without A23187 stimulation (Fig. 2D).

Increased levels of p-5-LO and p-ERK in neutrophils of Hypercholesterolemia-induced rats

To further elucidate the molecular mechanism underlying the translocation of 5-LO as a consequence of increased blood cholesterol levels, we next measured phosphorylation of 5-LO and the activity of ERK1/2- mediated signaling. Researchers have shown that the translocation 5-LO into the nucleus is accompanied by its phosphorylation by ERK1/2 (p-ERK1/2), whose activity depends on its own phosphorylation. Hence, we performed 
Fig. 3. Exposure to high cholesterol causes nuclear translocation of 5 - $\mathrm{LO}$ in vitro. (A): The production of LTB4 in neutrophils following incubation with the vehicle (control) and $\mathrm{M} \beta \mathrm{CD} /$ cholesterol complexes, with and without PD95809. (B): Western blot analysis of nuclear 5-LO in neutrophils incubated with the vehicle (control) and $\mathrm{M} \beta \mathrm{CD} /$ cholesterol complexes, with and without PD95809. (C): Western blot analysis of p-5-LO level in neutrophils incubated with the vehicle (control) and $\mathrm{M} \beta \mathrm{CD} /$ cholesterol complexes, with and without PD95809. (D): Western blot analysis of p-ERK1/2 in neutrophils incubated with the vehicle (control) and $\mathrm{M} \beta \mathrm{CD} /$ cholesterol complexes, with and without PD95809. Data are presented as Mean $\pm \mathrm{SD} ; \mathrm{n}=6$, duplicates. ${ }^{*} \mathrm{P}<0.05$; ${ }^{* *} \mathrm{P}<0.01$, NS: not significant; paired T- test. (E) Indirect immunofluorescent staining of 5-LO in neutrophils incubated with the vehicle (control) and M $\beta C D / c h o l e s-$ terol complexes, with and without PD95809 ( $\mathrm{n}=6$ independent experiments).

Western blot to analyze the levels of p-5-LO, ERK1/2 and p-ERK1/2. Although the expression of ERK1/2 was comparable between control and hypercholesterolemia rats, we found significantly elevated levels of both p-5-LO and p-ERK1/2 in the latter. This suggests that increased activity of ERK1/2, possibly leading to increased phosphorylation of 5-LO, is responsible for the enhanced nuclear distribution of 5-LO in response to increased blood cholesterol (Fig. 2E, F).

\section{Nuclear translocation of 5-LO in response to cholesterol exposure in vitro}

In order to further establish the direct relationship between cholesterol and 5-LO activity, we next used the $\mathrm{M} \beta \mathrm{CD} /$ cholesterol complexes to induce an upregulation of cholesterol levels in neutrophils in vitro. After incubating cells with the $\mathrm{M} \beta \mathrm{CD} /$ cholesterol complexes for $30 \mathrm{~min}$, we detected a significant increase in cholesterol content $(1.12 \pm 0.18$ versus $1.43 \pm 0.22 \mathrm{nmol} / 106 \mathrm{cells}, \mathrm{p}<0.05)$. In addition, the neutrophils isolated from control rats were incubated with either the vehicle, $\mathrm{M} \beta \mathrm{CD} /$ cholesterol complexes, or $\mathrm{M} \beta \mathrm{CD} /$ cholesterol complexes supplemented with $30 \mathrm{uM}$ ERK inhibitor PD98059 for $30 \mathrm{~min}$ at $37^{\circ} \mathrm{C}$. Next, we measured the levels of LTB4 and 5-LO (Western blot), the activity of 5-LO and ERK1/2 by performing Western blot for p-5-LO and p-ERK1/2, and the nuclear localization of 5-LO using indirect immunofluorescence. Consistent with previous results, LTB4 production was significantly increased in neutrophils exposed to $\mathrm{M} \beta \mathrm{CD} /$ cholesterol complexes (Fig. 3A). In addition, the levels of nuclear 5-LO (Fig. 3B, D), p-5-LO (Fig. 3C), and p-ERK1/2 (Fig. 3E) were significantly elevated in neutrophils incubated with the $\mathrm{M} \beta \mathrm{CD} /$ cholesterol complexes, 
in comparison to control cells. Furthermore, co- incubation with PD98059 suppressed all these alterations, providing additional evidence of the involvement of ERK1/2 signaling in cholesterol- mediated synthesis of LTB4.

\section{Discussion}

Our study demonstrates, both in vivo as well as in vitro, that hypercholesterolemia causes increased production of leukotriene B4 in neutrophils. Specifically, following LPS stimulation, the plasma LTB4 levels and their synthesis in neutrophils was significantly higher in hypercholesterolemia- induced rats, in comparison to the control group. This increase in LTB4 production was accompanied by increased phosphorylation of 5-lipoxygenase and its localization to the nucleus. Importantly, upregulation of LTB4 is also associated with enhanced phosphorylation, and hence activation, of ERK1/2. These observations suggest a mechanism that is consistent with findings from human and animal studies on the pathology of hypercholesterolemia- induced inflammation [12].

Our results are consistent with previous reports on the association between LTB4 production and blood cholesterol levels. In mast cells, pretreatment with $\mathrm{M} \beta \mathrm{CD}$ to decrease cholesterol levels, can dramatically reduce the synthesis of LTB4 in response to stimulation with A23187 [13]. Doi et al. found that hypercholesterolemia can increase LTB4 concentration in rat urine, and suggested it might directly contribute to accelerated hyperlipidaemic renal injury in these animals [14]. Das et al. reported a 2.3-fold increase in plasma LTB4 levels in ApoE-/- mice subjected to a six week hypercholesterol diet, when compared with animals that were fed control diet [15]. High fat diet was also shown to upregulate 5-LO expression in the retina of diabetic rats [16]. However, in contrast to these findings, Aleksandrov et al. showed that cholesterol sulfate and cholesterol phosphate inhibit the biosynthesis of LTB4 [17]. This might be due to physiologically relevant functional differences between cholesterol and its derivatives. Stanke-Labesque et al investigate the LTB4 production of neutrophil from obstructive sleep apnea patients or healthy control. They found thatLTB4 production were not correlated significantly with the cholesterol level. This may be due to the complex condition of patients. Although a multiple-linear regression analysis indicated that only apynea-hypoynea index was an independent predictor of LTB4 concentration, but this model merely explained $14 \%$ of the variance[18] . Maybe a specifical trial is needed to study LTB4 production.

In this study, we found increased nuclear localization of 5-LO in neutrophils of hypercholesterolemia- induced rats, irrespective of stimulation with its activator A23187. This is consistent with previous reports that suggest that nuclear localization of 5-LO increases its potential for subsequent synthesis of LTB4 [19]. Hence, we speculated that hypercholesterolemia- mediated increase in LTB4 production, might result from this mechanism. In neutrophils, LTB4 is primarily synthesized at the nuclear envelope, where activated cytosolic phospholipase A2 liberates arachidonic acid (AA) from membrane phospholipids. 5-LO interacts with FLAP, which facilitates the transfer of AA to 5-LO, for incorporation of molecular oxygen into the backbone of AA in a two-step reaction [4]. The expression and subcellular distribution of 5-LO, FLAP expression and availability of free AA, are key factors regulating LTB4 synthesis. Here, we found that while the levels of 5-LO, FLAP and AA remain unaltered in response to high cholesterol, the subcellular distribution of 5-LO is significantly modified. LTA4 hydrolase is the critical enzyme to catylyze the LTA4 into LTB4. Previous study found that LTA4 hydrolase activity in rats renal cortex were higher in the high cholesterol diet group than the normal diet group.LTA4 hydrolase may take part in the increased LTB4 production in hypercholesterolemia rats [14] . Specifically, both induction of hypercholesterolemia in vivo, as well as enrichment of the cholesterol content in neutrophils in vitro, led to increased phosphorylation and nuclear translocation of 5-LO. This was accompanied by enhanced LTB4 production. These findings support our 
hypothesis that hypercholesterolemia causes increased LTB4 production by stimulating nuclear translocation of 5-LO.

On further exploration of the mechanism underlying this phenomenon, we found that neutrophils isolated from hypercholesterolemic rats carried significantly higher level of p-ERK1/2, indicative of activated ERK1/2 signaling. ERK kinases and the subsequent signaling cascade are one of the major regulators of proliferation and cell growth in various cell types [20]. Previous research has shown that 5-LO can be phosphorylated by activated ERK1/2, followed by their transfer to the nucleus [21]. Since we also detected an increase in the levels of p-5-LO in response to hypercholesterolemia, this suggests that ERK1/2-mediated signaling might be responsible for the increased activity of 5-LO in response to high cholesterol. These findings are corroborated by our in vitro experiments where $\mathrm{M} \beta \mathrm{CD} /$ cholesterol complexinduced enrichment of cholesterol in neutrophils, led to similar changes. More importantly, we have shown that the ERK inhibitor PD98059 can block the upregulation in LTB4 in response to high cholesterol in vitro. This further supports our hypothesis that hypercholesterolemia activates ERK1/2 signaling and leads to nuclear translocation of 5-LO in neutrophils. This is consistent with a previous study where persistent activation of ERK1/2 was observed in vascular smooth muscle cells in a rabbit model of hypercholesterolemia [20]. Cholesterol has been shown to promote cell proliferation/migration in many cells. Cholesterol is mainly synthesised through 3-hydroxy-methylglutaryl CoA reductase / mevalonate pathway. The isoprenoids products of this pathway play vital roles in multiple cellular functions, including protein posttranslational modifications ,cell signaling, cell membrane integrity, cell cycle progression, and cholesterol synthesis [22]. As cholesterol is the main product , hypercholesterolemia may affect the pathway in feedback. Furthermore, cholesterol is a critical component of lipid rafts. Lipid rafts are dynamic microdomains enriching in many functional molecules, which have been demonstrated to regulate cell proliferation, apoptosis, migration, invasion and chemoresistance [23],hypercholesterolemia may regulate these function.

In conclusion, hypercholesterolemia can regulate subcellular localization of 5-LO and consequently, increase LTB4 production in neutrophils. Increased activity of the 5-LO/ LTB4 pathway could be one of the mechanisms responsible for hypercholesterolemiaassociated inflammation. Our findings further suggest that in patients who continue to have high (above guideline-mandated goals) levels of LDL-c despite receiving intensive statin therapy, targeting of the 5-LO/LTB4 pathway should be considered, in order to optimize the therapeutic strategy.

\section{Acknowledgement}

We thank Dr. Dong-Ying Zhang for assistance in preparation of figures and other helpful discussions. We thank Medjaden Bioscience Limited for assisting in the preparation of this manuscript. This work was supported by the National Natural Science Foundation of China (30900606).

\section{Disclosure Statement}

None declared.

\section{References}

Soehnlein O: Multiple roles for neutrophils in atherosclerosis. Circ Res 2012;110:875-888.

Drechsler M, Megens RT, van ZM, Weber C, Soehnlein 0:Hyperlipidemia-triggered neutrophilia promotes early atherosclerosis. Circulation 2010;122:1837-1845 
- 3 Stokes KY, Clanton EC, Clements KP, Granger DN: Role of interferon-gamma in hypercholesterolemiainduced leukocyte-endothelial cell adhesion. Circulation 2003;107:2140-2145.

4 Poeckel D, Funk CD: The 5-lipoxygenase/leukotriene pathway in preclinical models of cardiovascular disease. Cardiovasc Res 2010;86:243-253.

-5 Aiello RJ, Bourassa PA, Lindsey S, Weng W, Freeman A, Showell HJ: Leukotriene b4 receptor antagonism reduces monocytic foam cells in mice. Arterioscler Thromb Vasc Biol 2002;22:443-449.

-6 Stanke-Labesque F, Pepin JL, Gautier-Veyret E, Levy P, Back M: Leukotrienes as a molecular link between obstructive sleep apnoea and atherosclerosis. Cardiovasc Res 2014;101:187-193.

-7 Lee SJ, Choi EK, Seo KW, Bae JU, Kim YH, Park SY, Oh SO, Kim CD: 5-lipoxygenase plays a pivotal role in endothelial adhesion of monocytes via an increased expression of mac-1. Cardiovasc Res 2013;99:724733.

8 Cipollone F, Mezzetti A, Fazia ML, Cuccurullo C, Iezzi A, Ucchino S, Spigonardo F, Bucci M, Cuccurullo F, Prescott SM, Stafforini DM: Association between 5-lipoxygenase expression and plaque instability in humans. Arterioscler Thromb Vasc Biol 2005;25:1665-1670.

-9 Tardif JC, L PL, Ibrahim R, Gregoire JC, Nozza A, Cossette M, Kouz S, Lavoie MA, Paquin J, Brotz TM, Taub R, Pressacco J: Treatment with 5-lipoxygenase inhibitor via-2291 (atreleuton) in patients with recent acute coronary syndrome. Circ Cardiovasc Imaging 2010;3:298-307.

10 Pergola C, Dodt G, Rossi A, Neunhoeffer E, Lawrenz B, Northoff H, Samuelsson B, Radmark O, Sautebin L, Werz O: Erk-mediated regulation of leukotriene biosynthesis by androgens: A molecular basis for gender differences in inflammation and asthma. Proc Natl Acad Sci U S A 2008;105:19881-19886.

11 Oh H, Mohler ER, Tian A, Baumgart T, Diamond SL: Membrane cholesterol is a biomechanical regulator of neutrophil adhesion. Arterioscler Thromb Vasc Biol 2009;29:1290-1297.

12 Meng Z, Yan C, Deng Q, Dong X, Duan ZM, Gao DF, Niu XL: Oxidized low-density lipoprotein induces inflammatory responses in cultured human mast cells via toll-like receptor 4 . Cell physiol and biochem 2013;31:842-853.

13 You HJ, Seo JM, Moon JY, Han SS, Ko YG, Kim JH: Leukotriene synthesis in response to a23187 is inhibited by methyl- beta-cyclodextrin in rbl-2h3 cells. Mol Cells 2007;23:57-63.

- 14 Doi K, Hamasaki Y, Noiri E, Nosaka K, Suzuki T, Toda A, Shimizu T, Fujita T, Nakao A: Role of leukotriene b4 in accelerated hyperlipidaemic renal injury. Nephrology (Carlton) 2011;16:304-309.

15 Das R, Ganapathy S, Mahabeleshwar GH, Drumm C, Febbraio M, Jain MK, Plow EF: Macrophage gene expression and foam cell formation are regulated by plasminogen. Circulation 2013;127:1209-1218, e1201-1216.

-16 Mancini JE, Ortiz G, Croxatto JO, Gallo JE: Retinal upregulation of inflammatory and proangiogenic markers in a model of neonatal diabetic rats fed on a high-fat-diet. BMC ophthalmol 2013;13:14.

17 Aleksandrov DA, Zagryagskaya AN, Pushkareva MA, Bachschmid M, Peters-Golden M, Werz O, Steinhilber D, Sud GF: Cholesterol and its anionic derivatives inhibit 5-lipoxygenase activation in polymorphonuclear leukocytes and monomac6 cells. FEBS J 2006;273:548-557.

-18 Stanke-Labesque F, Pepin JL, de Jouvencel T, Arnaud C, Baguet JP, Petri MH, Tamisier R, Jourdil JF, Levy P, Back M: Leukotriene b4 pathway activation and atherosclerosis in obstructive sleep apnea. J Lipid Res 2012;53:1944-1951.

19 Brock TG, McNish RW, Bailie MB, Peters-Golden M:Rapid import of cytosolic 5-lipoxygenase into the nucleus of neutrophils after in vivo recruitment and in vitro adherence. J Biol Chem 1997;272:8276-8280.

$20 \mathrm{Hu}$ Y, Dietrich H, Metzler B, Wick G, Xu Q: Hyperexpression and activation of extracellular signal-regulated kinases (erk1/2) in atherosclerotic lesions of cholesterol-fed rabbits. Arterioscler Thromb Vasc Biol 2000;20:18-26.

21 Werz O, Burkert E, Fischer L, Szellas D, Dishart D, Samuelsson B, Radmark O, Steinhilber D: Extracellular signal-regulated kinases phosphorylate 5-lipoxygenase and stimulate 5-lipoxygenase product formation in leukocytes. FASEB J 2002;16:1441-1443.

22 Wang Z, Wu Y, Wang H, Zhang Y, Mei L, Fang X, Zhang X, Zhang F, Chen H, Liu Y, Jiang Y, Sun S, Zheng Y, Li N, Huang L: Interplay of mevalonate and hippo pathways regulates rhamm transcription via yap to modulate breast cancer cell motility. Proc Natl Acad Sci U S A 2014;111:E89-98.

23 Zou J, Xu L, Ju Y, Zhang P, Wang Y, Zhang B: Cholesterol depletion induces antxr2-dependent activation of mmp-2 via erk1/2 phosphorylation in neuroglioma u251 cell. Biochem Biophys Res Commun 2014;452:186-190. 